\title{
Lorenzo Renzi, Gli elfi e il Cancelliere. In Germania con Proust
}

\section{Gabriella Bosco}

\section{(2) OpenEdition \\ 1 Journals}

\section{Edizione digitale}

URL: http://journals.openedition.org/studifrancesi/4525

DOI: $10.4000 /$ studifrancesi. 4525

ISSN: 2421-5856

\section{Editore}

Rosenberg \& Sellier

\section{Edizione cartacea}

Data di pubblicazione: 1 settembre 2016

Paginazione: 388-389

ISSN: 0039-2944

\section{Notizia bibliografica digitale}

Gabriella Bosco, "Lorenzo Renzi, Gli elfi e il Cancelliere. In Germania con Proust », Studi Francesi [Online], 179 (LX | II) | 2016, online dal 01 septembre 2016, consultato il 18 septembre 2020. URL : http:// journals.openedition.org/studifrancesi/4525; DOI : https://doi.org/10.4000/studifrancesi.4525

Questo documento è stato generato automaticamente il 18 settembre 2020.

\section{(c) (i) $\odot$}

Studi Francesi è distribuita con Licenza Creative Commons Attribuzione - Non commerciale - Non opere derivate 4.0 Internazionale. 


\title{
Lorenzo Renzi, Gli elfi e il Cancelliere. In Germania con Proust
}

\author{
Gabriella Bosco
}

\section{NOTIZIA}

LORENZO RENZI, Gli elfi e il Cancelliere. In Germania con Proust, Il Mulino, Bologna, 2015, «Studi e Ricerche - Critica letteraria» 699, 264 pp.

1 «Studiare come Proust incontra il pensiero, la musica, l'arte della Germania e come i tedeschi leggono e recepiscono Proust è una pagina della storia della cultura d'Europa», scrive Lorenzo RENZI nella «Premessa» (pp. 7-11) di questo interessante volume.

2 Nella prima parte di esso (pp. 13-54), egli illustra come l'autore della Recherche abbia guardato alla Germania: la Germania paesistica della Nahe, affluente del medio Reno; la Germania rococò e romantica di Beethoven e di Wagner; la Germania, infine, in guerra con la Francia nel primo conflitto mondiale. Uno sguardo sempre simpatetico, lo definisce Renzi, come conferma la lettura ravvicinata delle breve corrispondenza tra Proust e Ernst Robert Curtius, scambio epistolare già più volte pubblicato ma qui per la prima volta interpretato (nell'Appendice al quarto capitolo, pp. 91-103).

3 Nella seconda parte del volume poi, la più lunga, l'A. studia come hanno interpretato i tedeschi la Recherche di Proust, osservando quanto importi sapere della loro ricezione se si pensa che essa fu la lettura più pronta, eccezionalmente precoce, e la più viva $\mathrm{e}$ profonda del capolavoro. Renzi offre quindi la sua penetrante lettura di un Proust non più visto come direttamente legato ai decadenti, a Huysmans e a Wilde, di cui poteva sembrare il continuatore, ma - secondo l'acuta intuizione di Erich Auerbach in Mimesis - "come creatore di una "vera epica dell'anima"», in cui «la concezione moderna del tempo interiore si collega alla concezione neoplatonica che l'immagine primigenia dell'oggetto si trovi nell'anima dell'artista; il quale, compreso egli stesso nell'oggetto, in veste di osservatore si è staccato da questo e affronta il proprio passato». Ed era del resto proprio Auerbach che, pur ammirando «l'inaudita preziosità del tessuto 
linguistico» della Recherche, non aveva esitato a definire «ripugnante [...] il fetore di una struttura sociale già in via di putrefazione». Renzi spiega qui che il critico si riferiva «certamente alla nobiltà francese rappresentata dalla società del Faubourg SaintGermain e particolarmente dai Guermantes, che occupa una buona parte del romanzo». Fa notare che in Proust l'aristocrazia non è raccontata «solo in innocue conversazioni davanti a una tazza di tè coi pasticcini», bensì osserva come l'intera opera sia costruita «attorno al contrasto tra la dissipazione della vita sociale e la sua redenzione nella letteratura», e dunque la sua trasformazione in opera d'arte. Già in un piccolo ma importante saggio del 2012, anch'esso edito dal Mulino, e intitolato Proust e Vermeer. Apologia dell'imprecisione, ragionando intorno al «petit pan de mur jaune», il dettaglio nella Veduta di Delft di fronte alla quale Bergotte muore, Renzi aveva messo in evidenza la distanza esistente, nella Recherche, fra la realtà e la sua rappresentazione, e aveva scritto: «Né in questo libro né in nessun altro è possibile riprodurre il muretto di Vermeer. Né si può vederlo al Mauritshuius dell'Aja, dove la Veduta di Delft è esposta. La ragione è che il muretto di Vermeer è diventato il muretto di Proust, un dettaglio della pittura olandese che esiste solo nella sua pagina, e che i nostri occhi non possono vedere». Tanto è vero che, secondo Renzi, Proust ha sovrapposto due particolari del quadro di Vermeer, un tettuccio inondato di sole ("giallo") nella zona interna destra della tela e due lunghi ma pallidi muri vicino a un ponte basculante, scambiato per una tettoia, all'estremità sempre destra dell'opera. Da queste osservazioni egli aveva dedotto la mancanza di esattezza di Proust, un tratto assolutamente non in contraddizione con il suo genio: anzi, scriveva allora, il genio di Proust si rivela proprio nella particolare deformazione che imprime alle cose, proprio come, in pittura, si potrebbe dire per esempio di El Greco, di Modigliani e di molti altri artisti.

Ora, in questo suo nuovo libro dedicato all'autore della Recherche, Renzi fa capire in che misura la comprensione della genialità di Proust sia passata per la lettura dei grandi della critica stilistica tedesca, che hanno dimostrato come Proust riesca a determinare le cose, imprimendo nella rappresentazione l'immagine allegorica del significato. Curtius e Spitzer, ci dice, hanno studiato lo stile linguistico in Proust come strumento di conoscenza e di rappresentazione della sua visione del mondo. «Lo stile è il modo che ha un autore di conoscere le cose», scriveva del resto Gianfranco Contini nei suoi Esercizi di lettura sopra autori contemporanei con un'appendice su autori non contemporanei (Parenti, Firenze, 1939). Dieci anni prima, Walter Benjamin, nel suo saggio Zum Bilde Prousts, già aveva imboccato questa strada riflettendo sul «culto [...] dell'analogia da parte di Proust», che aveva inventato una scrittura fondata sulla «somiglianza appassionata» fra le cose e fra le parole e le cose. Così Leo Spitzer, nei suoi Études de style del 1918 individuando in Proust l'«accoppiamento tra il formale e il semantico» oggi studiato da Renzi, osservava come in un passo che finisce, dopo straordinarie serie di associazioni, con una «chute d'eau», proprio la «chute d'eau» crei una magnifica «chute de phrase».

5 Ma anche Ortega y Gasset, in un saggio del 1923 che Renzi evoca per la sua vicinanza con la cultura tedesca, Le temps, la distance et la forme chez Proust pubblicato nel numero Hommage à Marcel Proust della «Nouvelle Revue Française» l'anno dopo la morte dello scrittore, aveva scritto che «per Proust è necessario essere prolisso e minuzioso per la semplice ragione che si avvicina agli oggetti più di quello che è comune. È stato l'inventore di una nuova distanza tra noi e le cose». E un grandissimo della critica europea, Ernst Robert Curtius, che per primo recensì la Recherche, in una lettera spedita a Proust dell'aprile 1922, dopo l'uscita di Sodome et Gomorrhe, gli aveva confessato: «La 
lettura del Suo libro è per me una delle più pure e più grandi gioie spirituali che mi siano state concesse negli ultimi anni. Lei ha dato nuovi motivi al mio amore per la Francia. Ammiro questa unione fra inesauribile ricchezza di vita e sovrana chiarezza intellettuale. Lei è riuscito a realizzare quello che Balzac definisce il carattere del genio: empreindre l'idée dans le fait». Nel saggio del 1925 Französischer Geist im neuen Europa (Deutsche Verlags-Anstalt), Curtius - innamorato dello «spirito francese» - dichiarava come da esso si aspettasse una spinta decisiva nel rinnovamento di quello europeo.

Dopo Curtius, Spitzer e Auerbach, Renzi si occupa nel quinto capitolo (pp. 107-133) di Victor Klemperer e affronta il tema della lettura marxista di Proust, presentando esponenti di diverse e addirittura convergenti concezioni del marxismo, alcune propense a condannare Proust tra i cascami del capitalismo e del decadentismo, altri a salvarlo. Una rassegna certamente non completa in cui figurano però due grossi nomi: Adorno, che si è confrontato molte volte con Proust, qui presentato attraverso un aforisma di Minima Moralia, e Manfred Naumann, il maggiore interprete di Proust della Germania orientale (capitolo 7, pp. 197-230).

7 L'ottavo e ultimo capitolo (pp. 231-242) è dedicato a Hans Robert Jauss, grandissimo romanista del secondo dopoguerra, visto anch'esso non per l'opera maggiore (Tempo e ricordo nella "Recherche" di Marcel Proust, 1986), ma per un aspetto meno noto: i parallelismi secondo Jauss tra le opere universali di Dante e Proust, enucleati in un lungo articolo di tre anni successivo. Il capitolo contiene anche una breve nota su un importante scrittore sempre del secondo dopoguerra, autore di un notevole saggio su Proust, Martin Walser.

8 L'idea alla base del volume di Renzi è che l'esegesi di un'opera sia favorita dalla lettura ragionata dei critici che ci hanno preceduti: «Ogni grande critico coglie e sviluppa una faccetta dell'opera d'arte, ed è l'insieme di tutte le faccette che forma la figura solida virtuale che è l'opera d'arte nella sua vita secolare». All'elaborazione del mosaico della critica proustiana, il saggio di Renzi contribuisce in maniera suadente. 